\title{
A Scoping Review Protocol to Map Empirical Evidence that Illuminates the Dark Side of Occupations Among Adults
}

\author{
Rebecca Twinley ${ }^{1 *}$, Leonie Boland ${ }^{2}$, Lisa Bunn $^{3}$, Gayle Letherby ${ }^{4}$ \\ ${ }^{1}$ School of Health Sciences, University of Brighton, Eastbourne, United Kingdom \\ ${ }^{2}$ Alumni Research Fellow, University of Plymouth, United Kingdom \\ ${ }^{3}$ Lecturer of Clinical Research and Programme Lead MClinRes, School of Health Professions, \\ University of Plymouth, United Kingdom \\ ${ }^{4}$ Visiting Specialist, School of Health Professions, University of Plymouth, United Kingdom
}

\begin{abstract}
The objective of this review is to explore existing literature to identify, map, and synthesise past accounts of occupations that could be considered as constituting the dark side of occupation and which could, consequently, be identified and discussed as such. Presenting findings through use of a synthesis matrix, and formulating a descriptive account of the types of occupations (including their form, function, meaning, and contribution to identity and becoming) that constitute the dark side of occupation, is anticipated to assist with prioritising future collaborative research endeavours, as part of an intended programme of research.
\end{abstract}

Specifically, the review questions are:

i) What past accounts of occupations have been discussed or explored in the literature that would constitute falling under the conceptual 'umbrella' of the dark side of occupation?

ii) What specific occupations that challenge the pervasive belief in the link between health and occupation have been discussed for the adult population, across all cultures?

iii) Where do gaps of knowledge remain regarding the less explored occupations people subjectively experience, and which indicate the priority research areas that need to be explored from an occupational perspective?

Keywords: adults, occupation, dark side of occupation, occupational science, healthcompromising, damaging, deviant

\section{Background}

Occupations are all of the things that individuals do in their lives every day (Sundkvist \& Zingmark, 2003) on their own and with others, such as their families and communities. Occupations are, therefore, all the things people need, want, and are expected to do (World Federation of Occupational Therapists, 2019) and are understood to impact upon, and be impacted by, our health and well-being.

Occupational science is the academic study of human occupation (Zemke \& Clark, 1996). From its inception in the late 1980s, occupational science was intended to provide an interdisciplinary theoretical and scientific perspective regarding humans as occupational

\footnotetext{
* Correspondence to Rebecca Twinley, University of Brighton, Robert Dodd Building, Darley Road, Eastbourne, BN20 7UR. Email: R.Twinley@Brighton.ac.uk

Social Science Protocols, March 2020, 1-13.

http://dx.doi.org/10.7565/ssp.2020.2807
} 
beings (Yerxa, 1993). Due to its focus on human behaviour, occupational science is considered a social science, just as anthropology, psychology, and sociology are (Yerxa, 1998). Occupational scientists have been theorising and conducting interdisciplinary research aimed at understanding the form (how an occupation is performed, or done), function (its purpose), meaning (how it is understood by the person performing or doing said occupation) of human occupation (Clark et al., 1991; Yerxa, 1990). Contributing to the substantial body of social science literature concerning the concept of identity (Vignoles, Schwartz, \& Luyckx, 2011), occupation is understood as a synthesis of doing, being, becoming, and belonging (Wilcock, 1999; Wilcock, 2006). That is, those things people all need and want to do (occupations) are a means through which they can develop and express their personal identities, and to achieve a sense of belonging (Ikiugu, 2005; Wilcock, 2006).

\section{The Dark Side of Occupation}

The concept of the 'dark side of occupation' was first theorised by Twinley in a coauthored paper (Twinley \& Addidle, 2012) (see also Twinley, 2013, Twinley, 2017). This was in response to Twinley's recognition that, across the world, occupational science, occupational therapy (and other, neighbouring health professions) have tended to focus on the links between occupation (the things people do in their daily lives), health, and wellbeing. Consequently, there is a significant disregard of occupations that may be harmful, unhealthy or health-compromising, damaging, deviant, destructive, anti-social, illegal, immoral. There is growing recognition that, beyond this, there has been minimal exploration of occupations that range from anything such as mundane or ordinary, to the atypical and extraordinary (publication pending). Naming the concept the 'dark side of occupation;' afforded Twinley the ability to challenge existing, fundamental beliefs, and to trigger debate and research activity in the area of less-explored occupations. This internationally recognised concept provides a conceptual lens for people (including but not exclusive to, occupational therapists) to use in their practice. It is now a widely accepted field of study with ever-growing international profile. For instance, in May 2019, Twinley became as Approved member of the Occupational Therapy Europe Register of Experts in the area of Dark Side of Occupation. Furthermore, there is increasing international discussion, evidenced by interdisciplinary events such as the Society for the Study of Occupation's (SSO: USA,(USA, 2019) 18th Annual Research Conference, which had the theme: 'The Darker Side of Occupations: Illegal, Taboo, Risky'.

The concept has interdisciplinary appeal and relevance, and has done from its inception, as evidenced by the first publication (Twinley \& Addidle, 2012) to document use of this term that was written in collaboration with a former colleague, a criminologist. Twinley suggested that in order for people's range of occupational needs to be met, the definition of occupation itself must be developed to reflect this, stating: "... the definition of occupation needs to include aspects of doing that are not deemed as prosocial, healthy or productive, including non-consensual or deviant sexual acts, drug misuse, alcohol misuse, violence and all other criminal activity" (Twinley \& Addidle, 2012). Since this initial publication, three further seminal pieces of work relating to the dark side of occupation have been published; two of these are written by the concept's creator $(2013,2017)$. A third article, which is very similar to the theme and aim of Twinley's 2013 paper, was written by a group of authors (Kiepek, Beagan, Rudman, \& Phelan, 2019) who cited Twinley's work in their paper as they state their preference for different terminology. Overall, these four papers have introduced some debate and all have concluded that in order for the understanding of occupations to broaden and deepen, evidence needs to be generated that explores occupations that have, until now, either had little to no examination specifically from an occupational perspective. 
Hence, initial validation of the research field and conceptual themes that emerged now requires further examination via research. Themes that align to the dark side of occupation are emerging in contemporary literature and exist in historical accounts, albeit under different and varying terminologies. Yet, to date, published accounts and research findings underpinning the dark side of occupation remain un-synthesised. To reach this point, there is a need to establish the absolute interdisciplinary relevance of the concept; the crucial step to doing so is to scope the literature to determine which occupations could be considered as constituting the dark side of occupation and which could, as a consequence of the scoping review, be identified and discussed as such. Therefore, this current scoping review is not only timely but also crucial for the development of the concept and subsequent related research endeavours.

The rationale for scoping accounts of occupations of adults is their significant difference in lived experience, and in their subjective experience of occupations, compared to childhood occupations (0-18). For instance, play is regarded as a key occupation in childhood (Moore, 2018) and, therefore, is a significant focus in the literature regarding childhood occupations. The rationale for excluding adults living or residing in an institution, in-patient setting, or care facility is that there is evidence to show adults living in certain contextual environments experience and engage in occupations in specific (contextually-driven or dependent) ways. For instance, Cunningham and Slade (2019) explored the lived experience of homelessness amongst five men, and found their engagement in occupation, whilst sleeping rough and later when residing in a homeless hostel, was centred around survival. Likewise, other work has found occupational engagement to be greatly impacted upon by environments, such as regional secure units, where security demands are priority and limitations are in place (Morris, Cox, \& Ward, 2016).

\subsection{Review objective/questions}

The objective of this review is to explore existing empirical evidence to identify, map, and synthesise past accounts of occupations that could be considered as constituting the dark side of occupation and which could, consequently, be identified and discussed as such. Presenting findings through use of a synthesis matrix, and formulating a descriptive account of the types of occupations (including their form, function, meaning, and contribution to identity and becoming) that constitute the dark side of occupation, is anticipated to assist with prioritising future collaborative research endeavours, as part of an intended programme of research.

The three questions of this review are: i) What past accounts of occupations have been discussed or explored in the literature that would constitute falling under the conceptual 'umbrella' of the dark side of occupation? ii) What specific occupations that challenge the pervasive belief in the link between health and occupation have been discussed for the adult population, across all cultures? iii) Where do gaps of knowledge remain regarding the less explored occupations people subjectively experience, and which indicate the priority research areas that needs to be explored from an occupational perspective?

\section{Methods/Design}

Initially guided by the Joanna Briggs Institute (JBI) (Peters et al., 2015) methodology for a recommended format, a standard scoping review framework will be used to conduct the review by an interdisciplinary study team of a sociologist, a physiotherapist, and two occupational scientists and therapists. The latter two members will independently screen records for inclusion using the criteria outlined in this protocol. 


\subsection{Inclusion criteria}

Eligibility criteria and methods of analysis have been determined a priori.

\subsection{Participants}

Where studies do include participants, this scoping review will consider articles that include adults aged 18 and over. As the main objective of this scoping review is to explore the dark side of occupation in adults, papers which relate to people under 18 years will be excluded. The term 'adult' will not be used as part of the search strategy as initial searches revealed how this significantly limits results. Where age is not stated, articles will still be considered if regarded to be a discussion relevant to any age of adult. Also to include all: sexes (refers to the biological, genetic and physiological processes that generally distinguish females from males) or genders, ethnic identities, educational backgrounds, socioeconomic backgrounds, sociocultural identities, health status, religious/spiritual beliefs.

\subsection{Concept}

The concept of interest is synthesising the literature that pertains to the dark side of occupation. Occupation itself is recognised in a broader sense than lay language would denote (as paid work), and can be defined as: "The experiences of humans which necessitate active engagement, have purpose and meaning, and are contextualized" (Molineux, 2017). This current review will consider all of the different ways, definitions, or classifications of occupation that have been stated in the four seminal pieces of work relating to the dark side of occupation; two of these are written solely by the concept's creator (Twinley, 2013; 2017), one was co-authored by Twinley (2012), and one article was written by a group of authors (Kiepek et al., 2019) who have cited Twinley's work in their paper. The latter paper is similar in theme and content to Twinley's 2013 article, and closely related to the concept of the dark side of occupation. To illustrate, the search strategy includes those key words - as highlighted in bold here - listed in the first published definition of the dark side of occupation: "Occupations that remain unexplored - such as those that are health compromising, damaging, and deviant - and which therefore challenge the pervasive belief in a causal relationship between occupation and health" (Twinley, 2017). Appendix II further shows how the key words were identified from the aforementioned literature.

\subsection{Context}

Articles will be considered for inclusion in this review within the context of studies conducted with adults in any country. Due to this, the search strategy (Appendix I) reveals that context has been left open to include all settings. In addition, those articles where it is revealed the participants are living or residing in an institution (such as a psychiatric unit, prison, or addiction centre), in-patient setting, or residential care, including hostels and shelters, at the time of data collection will be excluded.

\subsection{Types of sources}

Sources to be considered are described as falling within professional literature:

Professional literature:

- Peer reviewed, published papers and doctoral theses, as per JBI (2013) levels of evidence for meaningfulness 1-3

1. Qualitative or mixed-methods systematic review

2. Qualitative or mixed-methods synthesis

Social Science Protocols, March 2020, 1-13. 


\section{Single qualitative study}

- Papers exploring the lived experience of everything considered as the dark side of occupation. It does not necessarily need to be mentioned in the aim/objectives (does not have to be the primary research aim, nor does it need to be named as a secondary outcome).

- Studies that specifically focus on the subjective experience of occupation.

- All methodological approaches (where stated their approach), and designs as listed under the JBI levels of evidence for meaningfulness (due to seeking description of lived experience of occupations and/or descriptive information regarding occupations).

- Full-text papers published in English, in the last ten years (since 2009) in an effort to align with the emergence of relevant contemporary literature, and that which has been published around contemporary paradigms and developments.

Sources that will not be considered:

- This scoping review will not include books, book chapters, conference proceedings, or grey literature, which will allow us to map how the dark side of occupation is conceptualised and explored in original research and expert opinion pieces.

\subsection{Search strategy}

The search strategy will aim to find published literature. A three-step approach will be taken, in line with JBI recommended methodology (Peters et al., 2015).

i) The initial step included a limited search of PubMed (MEDLINE) and CINAHL and analysis of the text words contained in the title and abstract, and of the index terms used to describe the article. This has informed the development of the search strategy - a revised example of which has been appended (Appendix I). To elucidate, the first three CINAHL searches generated: 1) 2,208,760, 2) 6,987, 3) 5,271 results, respectively (see Appendix III for search strategy). Because the initial searches generated an unmanageable number of results, the team returned to considering search terms and agreed to draw upon the four key pieces of literature from occupational therapy and science publications that have introduced the issue of occupations that have been left in the dark, silenced, and unexplored. These articles were referred to and key terms identified (Appendix II) which are the terms used in the final search strategy (Appendix I). Following this strategy, the first CINAHL search generated 1407 results.

ii) Secondly, therefore, these terms will be used to search within the electronic databases identified below. It is accepted that an iterative approach has been needed in order to confirm the search strategy.

iii) Thirdly, a search of reference lists in all included articles will then performed to search for any additional items. While an historical consideration of the concept would be interesting, the research team agreed that literature published in the last decade, since 2009 , would be included as the content and findings are deemed to have more relevance to contemporary practice, education, and research. Only English language materials will be included as the author and research assistant do not have the resources for translation. A record will be kept of the number of papers excluded on the basis of language and reported in the PRISMA flow diagram. 


\subsubsection{Information sources}

The databases to be searched include: CINAHL, MEDLINE (EBSCO host), AMED, Embase, PsycINFO, SocINDEX, Scopus and OTSeeker. All professions and disciplines will be included. That said, as the concept of the dark side of occupation stemmed from the identification of a gap in examining occupations that are not necessarily healthy (for instance), a specific search within the Journal of Occupational Science will be performed.

\subsection{Study selection}

Following the search, all identified citations will be imported into EndNote X8 (Clarivate Analytics, PA, USA). At this stage any duplicate citations will be removed. Then all citations will be imported into the free, online application, Rayyan (QCRI, Qatar Computing Research Institute, USA), to allow for title and abstract blind screening. The titles and abstracts of citations will be screened independently by the author and the research assistant (the two reviewers) and compared to the inclusion criteria for the review. Publications identified as potentially relevant will be retrieved in full and their citation details will be imported into Rayyan. The full text of these selected citations will be fully assessed against the inclusion criteria for review independently by the two reviewers. Any arising disagreements between the reviewers at each stage of the study selection process will be resolved through consensus, or through utilising the third or fourth author as a third reviewer to decide. Publications that do not meet the inclusion criteria will be excluded and reasons for their exclusion will be reported in the scoping review. The results of the search will be reported in full in the final report and they will be presented in a PRISMA flow diagram (Moher, Liberati, Tetzlaff, Altman, \& The, 2009).

\subsection{Data extraction}

Results will be extracted through all data that is relevant and that informs the overarching research question and objectives. Tables will be used to chart data. The main results will be structured, based on the dark side of occupation concept that underpins the review, and supported by a descriptive summary. A data extraction tool has been developed specifically for this scoping review (Appendix IIII), and will be used to extract the relevant data from each paper. This follows more typical data extraction designs, and also utilises the work of occupational scientists who seek to understanding an occupation's form, function, meaning and contribution to identity and becoming. Indeed, this utilises two of the first approaches used in occupational science in order to study human occupation (Wilcock, 1999; Zemke \& Clark, 1996).

\section{Presentation of Results}

The extracted data may be presented using, but not limited to: tables, diagrams, figures, citation maps, word clouds, concept network maps. This will include a synthesis matrix of those occupations that may (conceptually) be considered as the dark side of occupation, presented in the form of a data table. This has been developed for this scoping review (Appendix V), however it is anticipated that this will be further refined for use during the review process. In subsequent publications or presentations of the final scoping review, this synthesis matrix will be presented as a work in progress, rather than an absolute or strict and final synthesis. A narrative summary will accompany any tabulated and/or charted results and this will describe how the results relate to the review objectives. A discussion of the data and the significance of the findings will be given in the final report. 


\section{Discussion}

The strengths of this proposed scoping review are that it presents an interesting topic and a concept that is gaining international, interdisciplinary attention (evidence by online impact and the forthcoming publication of a monograph of international perspectives regarding the dark side of occupation, edited by Twinley). In addition, this review is intended to make an important and unique contribution to the interdisciplinary occupational science research, as well as to related interdisciplinary practice such as occupational therapy.

The limitations of the proposed study lay chiefly in the fact that the dark side of occupation is a concept about such a vast array of human occupations that remain unexplored, or under-explored. Therefore, this protocol could be considered as too broad in scope. However, the review team remain confident that the work they have done to reach the point of agreeing on the final search criteria has been necessary and has led to considerable clarity.

\section{Declarations}

Competing interests: The authors declare that they have no competing interests.

Funding: This study is supported by pump priming awarded in response to the University of Plymouth Institute of Health and Community (IHC) Pump-priming call 2019/20.

\section{References}

Clark, F. A., Parham, D., Carlson, M. E., Frank, G., Jackson, J., Pierce, D., . . Zemke, R. (1991). Occupational science: Academic innovation in the service of occupational therapy's future. American Journal of Occupational Therapy, 45(4), 300-310. doi:10.5014/ajot.45.4.300

Ikiugu, M. N. (2005). Meaningfulness of occupations as an occupational-life-trajectory attractor. Journal of Occupational Science, 12(2), 102-109. doi:10.1080/14427591.2005.9686553

Institute, J. B. (2013). JBI levels of evidence 5. Retrieved from https://joannabriggs.org/sites/default/files/2019-05/JBI-Levels-of-evidence_2014_0.pdf

Kiepek, N. C., Beagan, B., Rudman, D. L., \& Phelan, S. (2019). Silences around occupations framed as unhealthy, illegal, and deviant. Journal of Occupational Science, 26(3), 341353. doi:10.1080/14427591.2018.1499123

Moher, D., Liberati, A., Tetzlaff, J., Altman, D. G., \& The, P. G. (2009). Preferred reporting items for systematic reviews and meta-analyses: The PRISMA statement. PLOS Medicine, 6(7), e1000097. doi:10.1371/journal.pmed.1000097

Molineux, M. (2017). A dictionary of occupational science and occupational therapy. Retrieved from https://www.oxfordreference.com/view/10.1093/acref/9780191773624.001.0001/acref9780191773624-e-0358.

Moore, A. (2018). Play and play occupation: a survey of paediatric occupational therapy practice in Ireland. Irish Journal of Occupational Therapy, 46(1), 59-72. doi:10.1108/IJOT-08-2017-0022

Morris, K., Cox, D. L., \& Ward, K. (2016). Exploring stories of occupational engagement in a regional secure unit. The Journal of Forensic Psychiatry and Psychology, 27(5), 684697. doi:10.1080/14789949.2016.1187759 
Peters, M. D., Godfrey, C., McInerney, P., Baldini Soares, C., Khalil, H., \& Parker, D. (2015). Chapter 11: Scoping reviews. Joanna Briggs Institute Reviewer's Manual. Retrieved from https://reviewersmanual.joannabriggs.org/

Sundkvist, Y., \& Zingmark, K. (2003). Leading from intermediary positions: first-line administrators' experiences of their occupational role and situation. Scandinavian Journal of Occupational Therapy, 10(1), 40-46.

Twinley, R. (2013). The dark side of occupation: A concept for consideration. Australian Occupational Therapy Journal, 60(4), 301-303. doi:10.1111/1440-1630.12026

Twinley, R. (2017). The dark side of occupation. In K. Jacobs \& N. MacRae (Eds.), Occupational therapy essentials for clinical competence (pp. 29-36). Thorofare, NJ: SLACK Incorporated.

Twinley, R., \& Addidle, G. (2012). Considering violence: The dark side of occupation. British Journal of Occupational Therapy, 75(4), 202-204. doi:10.4276/030802212x13336366278257

Society for the Study of Occupation: USA (2019, October 3-5). Proceedings of SSO: USA Annual Research Conference: The darker side of occupations: Illegal, taboo, risky, 7, 5051. Retrieved from: https://www.sso-usa.org/assets/docs/proceedings_2019.pdf

Vignoles, V. L., Schwartz, S. J., \& Luyckx, K. (2011). Introduction: Toward an integrative view of identity. In Handbook of identity theory and research, Vols. 1 and 2 (pp. 1-27).

New York, NY: Springer Science + Business Media.

Wilcock, A. A. (1999). Reflections on doing, being and becoming. Australian Occupational Therapy Journal, 46(1), 1-11. doi:10.1046/j.1440-1630.1999.00174.x

Wilcock, A. A. (2006). An occupational perspective of health. Thorofare, NJ: SLACK Incorporated

World Federation of Occupational Therapists. (2019). About occupational therapy. Retrieved from https://www.wfot.org/about-occupational-therapy

Yerxa, E. J. (1990). An introduction to occupational science: A foundation for occupational therapy in the 21st century. Occupational Therapy in Health Care, 6(4), 1-17. doi:10.1080/J003v06n04_04

Yerxa, E. J. (1993). Occupational science: A new source of power for participants in occupational therapy. Journal of Occupational Science, 1(1), 3-9. doi:10.1080/14427591.1993.9686373

Yerxa, E. J. (1998). Occupational science: a renaissance of service to humankind through knowledge. Occupational Therapy International, 7(2), 87-98.

Zemke, R., \& Clark, F. A. (Eds.). (1996). Occupational science: The evolving discipline. Philadelphia, Pa, USA: F.A. Davis Company. 


\section{Appendices}

\section{Appendix I: Search strategy $\left(2^{\text {nd }}\right.$ version $)$}

PubMed (MEDLINE) and CINAHL. Population-Concept: Search terms to include:

\begin{tabular}{|c|c|}
\hline Search & Query \\
\hline $\begin{array}{l}\# 1 \\
\text { Population }\end{array}$ & Left open \\
\hline $\begin{array}{l}\# 2 \\
\text { Concept }\end{array}$ & $\begin{array}{l}\text { occupation* } \\
\text { AND } \\
\text { abnormal; addict* anti-social; crime; criminal; damaging; dark side; disruptive; } \\
\text { deviant; extreme; harmful; health-compromising; illegal; immoral; inappropriate; non- } \\
\text { health-giving; non-health-promoting; non-consensual; non-sanctioned; shadows; } \\
\text { violen*; unacceptable; undesired; unexplored; unhealthy; unproductive }\end{array}$ \\
\hline $\begin{array}{l}33 \\
\text { Context }\end{array}$ & Left open to include all settings \\
\hline \#4 & $\# 1$ AND \#2 \\
\hline $\begin{array}{l}\text { Screening } \\
\text { factors }\end{array}$ & $\begin{array}{l}\text { Not institutional residents (homeless, refugee, or residents of care, psychiatric, or } \\
\text { judiciary institutions) } \\
\text { Published in last ten years (since 2009) }\end{array}$ \\
\hline
\end{tabular}




\section{Appendix II: Identifying search terms}

Twinley (and Addidle) 2012

\section{Considering violence: the dark side of occupation}

Arguably, some occupations may not promote health or wellbeing, such as violence, which is seen as harmful, disruptive and therefore 'antisocial'. How then can occupational therapists work from a truly occupational perspective without an understanding of such antisocial occupations? It is suggested that the definition of occupation needs to include aspects of doing that are not deemed as prosocial, healthy or productive, including nonconsensual or deviant sexual acts, drug misuse, alcohol misuse, violence and all other criminal activity.

\section{Twinley 2013:}

Amongst other things, it includes tasks, activities, routines or acts that are considered antisocial, perhaps even criminal and illegal. Use of the term 'dark side' is not intended to portray occupation as having two sides. As the definition and understanding of occupation has evolved, the great majority of accounts do now assert that occupation is something that is complex and multidimensional. It is certainly not something that can be divided into this side and that. However, in many ways the term 'dark side' seems fitting; it suggests occupation is something that has aspects which are less acknowledged, less explored and less understood. It presents occupation as something which has aspects to it that have been left in the shadows. Something that, when prompted to consider, we all know is there, yet something that many of us have not incorporated into our theory, understanding and use of occupation. Perhaps this is because there is an immense dearth of work that clearly incorporates those other aspects of occupation that could be seen to exist as part of the dark side. That is, occupations that could be one of, or a combination of, the following: anti-social; criminal; deviant; violent; disruptive; harmful; unproductive; non-health-giving; non-health-promoting; addictive and politically, socially, religiously or culturally extreme. Occupations that, to the individual performing them, could still be any combination of the following: meaningful, purposeful, creative, engaging, relaxing, enjoyable, entertaining, that can provide a sense of wellbeing and even that are occupational in the sense of being an individual's paid or unpaid work.

\section{Twinley 2017:}

Dark side of occupation: Occupations that remain unexplored-such as those that are health compromising, damaging, and deviant — and which therefore challenge the pervasive belief in a causal relationship between occupation and health.

\section{Kiepek 2019:}

Silences around occupations framed as unhealthy, illegal, and deviant We suggest the term "non-sanctioned occupations" to encompass occupations that, within historically and culturally bound contexts, tend to be viewed as unhealthy, illegal, immoral, abnormal, undesired, unacceptable, and/or inappropriate. 


\section{Appendix III: Search strategy $\left(1^{\text {st }}\right.$ version $)$}

\section{PubMed (MEDLINE) and CINAHL}

\section{Population (working-age adults)}

[adult*; client*; group*; individual; patient; people; person; resident; working-age; young person]

AND

\section{Concept (dark side of occupation)}

[activit*; adapt*; ADL OR ADLs; act; action; being; belong*; daily; do; doing; endeavour; engag*; enterprise; ergotherapie; everyday; function*; habit*; hobby; interest; job; leisure; life; livelihood; living; need*; occupation; occupational therap*; OR OT; occupational science OR OS; participat*; pastime; perform*; product*; role*; self-care; vocation; want; work]

AND

[banned; barred; bent; dark side; career; corrupt*; crime; criminal; crooked; cultur*; damage*; danger*; dare; daring; dark side; depriv*; destruct*; detriment*; deviant; develop*; devious; difficult*; dishonest; disparaging; disrupt*; distasteful; dodgy; dubious; expect*; forbidden; fraud*; ghoulish; grave; grievous; harm*; hazard*; health*; hurt*; illegal; illegitimate; illicit; illness; injurious; insalubrious; legislati*; mental; messy; minority; negative*; noxious; off-limits; offensive; offend*; outlaw*; perilous; physical; poisonous; precarious; prohibit*; profession; proscribed; pursuit; restrict*; resilience; risk*; satisfied; satisfaction; serious; shady; shifty; social; surviv*; suspicious; taboo; task*; threat*; toxic; treacherous; unhealthy; underhanded; unnatural; unlawful; unmentionable; unproductive; unsafe; unsanitary; unthinkable; unwholesome; wellbeing; wellness; wounding]

\section{Context (community-dwelling or living)}

[accommodat*; communit*; community based; community-dwelling; community dwelling community-living; community-living; domiciliary; environment; home; house*; setting*; therap*;

\section{Screening factors:}

Institutional residents (homeless, refugee, or residents of care, psychiatric, or judiciary institutions). 


\section{Appendix IIII: Data extraction tool}

\begin{tabular}{|l|l|}
\hline Author/s & \\
\hline Year of publication & \\
\hline Origin/country & \\
\hline Source & \\
\hline Aim/purpose (if stated or described) & \\
\hline $\begin{array}{l}\text { Population and size (where applicable) } \\
\text { including demographic factors }\end{array}$ & \\
\hline Context (if stated or described) & \\
\hline $\begin{array}{l}\text { Terms or language used to define or describe or } \\
\text { discuss the occupation/s (if given) }\end{array}$ & \\
\hline $\begin{array}{l}\text { Definition or description of the occupation/s (if } \\
\text { given) form/s, function/s, meaning/s }\end{array}$ & \\
\hline $\begin{array}{l}\text { Description of the occupation/s (if given) link to } \\
\text { identity and becoming }\end{array}$ & \\
\hline $\begin{array}{l}\text { Key findings/results/recommendations (if stated } \\
\text { or described) }\end{array}$ & \\
\hline $\begin{array}{l}\text { Implications for occupational therapy, } \\
\text { occupational science, or relating to an } \\
\text { occupational perspective (where applicable) }\end{array}$ & \\
\hline $\begin{array}{l}\text { Recommendations for further study or focus (if } \\
\text { stated or described) }\end{array}$ & \\
\hline
\end{tabular}

Social Science Protocols, March 2020, 1-13. 
Appendix V: Example synthesis matrix for topic: The dark side of occupation

\begin{tabular}{|c|c|c|c|c|}
\hline Occupation & Source & Context & $\begin{array}{l}\text { Form (what, how, where } \\
\text { and who, the observable } \\
\text { aspects, how occupation } \\
\text { is done/performed) } \\
\text { - Function (its purpose, } \\
\text { outcome, contribution to } \\
\text { development, health, } \\
\text { life) } \\
\text { - Meaning (the subjective } \\
\text { experience and how it is } \\
\text { understood by the doer, } \\
\text { motives) } \\
\text { Identity and becoming } \\
\text { (contribution to } \\
\text { formation or expression } \\
\text { of identity) }\end{array}$ & $\begin{array}{l}\text { Terms or } \\
\text { language used } \\
\text { to define or } \\
\text { describe or } \\
\text { discuss the } \\
\text { occupation/s }\end{array}$ \\
\hline $\begin{array}{l}\text { Alcohol } \\
\text { use* }\end{array}$ & $\begin{array}{l}\text { Wasmuth, } \\
\text { Crabtree, } \\
\text { \& Scott } \\
(2014)\end{array}$ & $\begin{array}{ll}\text { - Alcoholics } \\
\text { Anonymo } \\
\text { us } \\
\text { attendees } \\
\text { in the UK } \\
\text { - Six } \\
\text { women } \\
\text { and four } \\
\text { men } \\
24 \text { to } 75 \\
\text { years old }\end{array}$ & $\begin{array}{l}\text { - } \text { Alcohol as primary addiction } \\
\text { - } \quad \text { Survival } \\
\text { - } \quad \text { Contal stability } \\
\text { - } \text { On a continuum of the } \\
\text { individual's own control } \\
\text { - Penetration and pervasive (to } \\
\text { varying degrees) to one's life } \\
\text { - } \text { Identity and means the self- } \\
\text { identified } \\
\text { - } \quad \text { Motivation } \\
\text { - } \text { Haping/escape } \\
\text { - } \text { as habituation as routine and/or } \\
\text { Part of who I am, something } \\
\text { inherent, inseparable, and self- } \\
\text { defining }\end{array}$ & $\begin{array}{ll}\text { - } & \text { Addiction- } \\
\text { as- } \\
\text { occupation } \\
\text { - Substance- } \\
\text { related and } \\
\text { addictive } \\
\text { disorders }\end{array}$ \\
\hline
\end{tabular}

*Exemplar demonstrated for alcohol use only 